\title{
(2) OPEN ACCESS \\ Systemic arterial blood pressure and intracerebral hemorrhage after mechanical thrombectomy in anterior cerebral circulation
}

\author{
Jiaqi An, ${ }^{1}$ Yonglan Tang, ${ }^{1}$ Xiangqi Cao, ${ }^{1}$ Huijie Yuan, ${ }^{1}$ Meng Wei, ${ }^{1}$ Xingyun Yuan, \\ Aifeng Zhang, ${ }^{2}$ Yongxin Li, ${ }^{3}$ Ardan Saguner, ${ }^{4}$ Guoliang Li $\odot{ }^{5},{ }^{5}$ Guogang Luo ${ }^{1}$
}

\begin{abstract}
- Additional material is published online only. To view, please visit the journal online (http://dx.doi.org/10. 1136/jim-2020-001554).
\end{abstract}

For numbered affiliations see end of article.

\section{Correspondence to}

Dr Guoliang Li, First Affiliated Hospital of Xi'an Jiaotong University, Xi'an, China;

liguoliang_med@163.com and Professor Guogang Luo, Department of Neurology, The First Affiliated Hospital of Xi'an Jiaotong University, Xi'an, China;

lguogang@163.com

$\mathrm{JA}$ and $\mathrm{YT}$ contributed equally.

$J A$ and $Y T$ are joint first authors.

Accepted 5 February 2021 Published Online First 2 March 2021

\section{ABSTRACT}

The relationship between systemic arterial blood pressure (BP) and intracerebral hemorrhage (ICH) after mechanical thrombectomy (MT) of the cerebral artery remains unclear. This study aimed to determine the effect of $\mathrm{BP}$ variables on $\mathrm{ICH}$ after MT in patients with acute occlusions of the anterior cerebral circulation. Patients undergoing MT due to acute occlusions of the anterior cerebral circulation were enrolled in this singlecenter study. Non-invasive BP data following MT were obtained within the first 24 hours, including mean, maximum, minimum, difference between maximum and minimum, SD and coefficient of variation for systolic BP (SBP) and diastolic BP (DBP) and mean arterial pressure. ICH was defined and classified according to the European Cooperative Acute Stroke Study-II. In 164 enrolled patients (median age 65 (IQR 5675) years; $31.7 \%$ female), higher maximum ( 89.5 $\mathrm{mm} \mathrm{Hg}$ vs $98.5 \mathrm{~mm} \mathrm{Hg}, \mathrm{p}=0.001)$ and SD (9.8 $\mathrm{mm} \mathrm{Hg}$ vs $10.9 \mathrm{~mm} \mathrm{Hg}, p=0.038$ ) of DBP were associated with higher risk of ICH. The optimal cut-off values associated with $\mathrm{ICH}$ for maximum SBP were $155 \mathrm{~mm} \mathrm{Hg}$ and for maximum DBP $92.5 \mathrm{~mm} \mathrm{Hg}$, respectively. Higher BP within 24 hours after MT in acute occlusions of the anterior cerebral circulation is associated with a greater risk of $\mathrm{ICH}$. More studies are needed to further determine optimal BP goals in the acute phase after MT.

\section{INTRODUCTION}

Endovascular thrombectomy (EVT) has been proven beneficial to patients who had acute ischemic stroke (AIS) due to large vessel occlusion (LVO). ${ }^{1-5}$ The DAWN and DEFUSE-3 trials, designed to show the efficacy of thrombectomy in selected patients with occlusion of proximal vessels in the anterior cerebral circulation up to 24 hours after suspected onset of symptoms, promote further development of EVT. $^{6}$ However, intracerebral hemorrhage (ICH), as one of the common and potentially more serious complications after mechanical thrombectomy (MT), usually causes an increase in disability and mortality. Systemic arterial hypertension is an important risk factor for

\section{Significance of this study}

What is already known about this subject?

- The association between blood pressure (BP) and intracerebral hemorrhage (ICH) after endovascular thrombectomy (EVT) in patients who had acute ischemic stroke (AIS) has been controversial.

- Previous studies found higher maximum systolic BP (SBP) and SBP fluctuation after EVT to be associated with worse outcomes and higher rates of ICH in patients who had AIS, but only few studies reported the association between diastolic BP (DBP) and outcomes in patients who had AIS after EVT.

\section{What are the new findings?}

- Higher maximum DBP within 24 hours after mechanical thrombectomy (MT) was associated with higher odds of ICH (OR 1.03, $95 \% \mathrm{Cl} 1.01$ to 1.05 , adjusted $\mathrm{p}=0.028$ ) when adjusted for age, baseline National Institutes of Health Stroke Scale (NIHSS) score, baseline Glasgow Coma Scale score, recanalization status (successful defined as modified Thrombolysis in Cerebral Ischemia ( $\mathrm{mTICl}$ ) $2 b-3$ vs unsuccessful defined as mTICI $0-2 a$ ) and procedure time.

- A maximum SBP of $>155 \mathrm{~mm}$ Hg yielded an unadjusted OR of 2.53 (1.31-4.89) $(\mathrm{p}=0.006)$ and an adjusted OR of 2.42 (1.204.87) $(p=0.013)$ for suffering $\mathrm{ICH}$, and patients with a peak DBP $>92.5 \mathrm{~mm} \mathrm{Hg}$ had 4.56 times higher odds of ICH.

- For maximum DBP, an ideal cut-off value of $92.5 \mathrm{~mm} \mathrm{Hg}$ was identified ( $80 \%$ sensitivity, $57 \%$ specificity, area under the curve 0.65 , $95 \% \mathrm{Cl} 0.57$ to $0.74, \mathrm{p}=0.001)$.

ischemic stroke and $\mathrm{ICH}^{7-9}$ Although proper blood pressure (BP) management is associated with a reduced risk of ICH and mortality, ${ }^{10}$ the optimal target of BP management during and after MT remains unclear.

Current American Heart Association/American Stroke Association guidelines recommend maintaining BP at $\leq 180 / 105 \mathrm{~mm} \mathrm{Hg}$ for $24-48$ hours after $\mathrm{MT}^{11}$; however, it is 


\section{Significance of this study}

How might these results change the focus of research or clinical practice?

- Higher systemic arterial BP, especially maximal DBP, within 24 hours after MT in acute occlusions of the anterior cerebral circulation is associated with a greater risk of $\mathrm{ICH}$, regardless of age, admission NIHSS score, recanalization time and recanalization status.

- In order to prevent ICH, doctors should pay more attention to both SBP and DBP after MT.

recommended indiscriminately to all patients receiving MT. Intensive BP control, theoretically, could be beneficial to patients with successful MT in terms of risk of ischemic/ reperfusion hemorrhage. ${ }^{12}$ On the other hand, for patients with incomplete recanalization, permissive hypertension may be beneficial to maintain brain perfusion pressure.

Reasonable BP management after MT is considered helpful in preventing ICH; however, there are no consistent recommendations on specific BP management after MT. Some studies have found a correlation between hypertension and ICH after MT, ${ }^{13}$ while others reported no such association. ${ }^{10}{ }^{14}$ This study therefore aimed to investigate the association between per interventional BP and hemorrhagic transformation after MT in patients who had ischemic stroke.

\section{MATERIALS AND METHODS Patient population}

This is a retrospective, observational study of patients who had AIS ${ }^{15}$ with LVO of the anterior cerebral circulation undergoing MT. All consecutive unselected patients who were treated with MT between June 2015 and December 2019 in the stroke center of the First Affiliated Hospital of Xi'an Jiaotong University were enrolled in this study. Medical history, demographic information, baseline characteristics, initial imaging, and angiographic results of each case were obtained.

The protocol for selecting patients for MT and other stroke management decisions were made by the attending medical providers and the stroke care team according to current guidelines. Patients with premorbid modified Rankin Scale (mRS) score $>2$, severe systemic diseases such as terminal cancer, dementia, and end-stage heart or liver failure, and without a repeat head CT scan within 24 hours after MT were excluded. MT procedures were conducted using both stent retrievers and aspiration catheters. All patients were admitted to an intensive stroke unit for postprocedural care.

\section{Baseline demographic and treatment parameters}

Baseline parameters included age, sex, comorbidities, baseline mRS score, admission National Institutes of Health Stroke Scale (NIHSS) score, admission Glasgow Coma Scale (GCS) score, symptom onset-to-groin puncture time (minutes), pretreatment with intravenous tissue-type plasminogen activator (tPA) and admission systemic arterial BP levels. Procedural parameters included use of intra-arterial
tPA or tirofiban, modified Thrombolysis in Cerebral Ischemia (mTICI) score and procedure time.

Successful reperfusion was defined as $\mathrm{mTICI} \geq 2 \mathrm{~b}$ at the end of intervention. If MT of the targeting artery was not successful, rescue therapies, such as balloon angioplasty, stent implantation, intra-arterial thrombolysis, or intracatheter tirofiban administration, were performed. Head CT scans were regularly performed 24 hours after the procedure or whenever an ICH was suspected by clinical symptoms.

\section{BP parameters}

Non-invasive BP values before and after the procedure were recorded in the intervention report. Hourly BP during the first 24 hours was measured by a non-invasive BP cuff of appropriate size. All BP data were reviewed by trained clinicians based on guidelines for every patient after the MT procedure. BP parameters included mean, coefficient of variation, maximum and minimum systolic BP (SBP), maximum and minimum diastolic BP (DBP), and mean arterial pressure during the first 24 hours after MT. In addition, we included SBP and DBP ranges (maximum-minimum) and SD as parameters for BP variation.

\section{Definition of ICH after MT}

ICH was defined and classified as one of the following subtypes according to the European Cooperative Acute Stroke Study-II (ECASS-II) $^{16}$ : hemorrhagic infarction (HI)-1: small petechiae along the margins of the infarct; HI-2: more confluent petechiae within the infarcted area, but without space-occupying effect; parenchymal hematoma (PH)-1: blood clot not exceeding 30\% of the infarcted area with some mild space-occupying effect; and $\mathrm{PH}-2$ : dense blood clot(s) exceeding $30 \%$ of the infarct volume with significant space-occupying effect.

\section{Statistical analysis}

All statistical analyses were performed using SPSS V.24.0 software. Discrete variables are presented as counts (with percentages) and continuous variables as mean (SD) or median (IQR), as appropriate. Normal distribution of data was tested using the Shapiro-Wilk test. A Kruskal-Wallis test was used for non-parametric tests. Student's t-test and Kruskal-Wallis test were used to compare BP parameters at the univariate level, and multivariable logistic regression analyses were used to evaluate parameters of SBP and DBP as possible predictors of $\mathrm{ICH}$. Variables with $\mathrm{p}<0.05$ on univariate analysis were included in the logistic regression models. Each BP variable that was significant at the univariate level or that was considered clinically relevant was entered into the multivariable logistics regression model. The area under the receiver operating characteristic curve has been used to determine the optimal cut-off BP values, which were defined as values with maximal Youden index. All tests used a two-sided $\alpha$ level of 0.05 for significance. All effect sizes are reported with $95 \% \mathrm{CI}$ in addition to $\mathrm{p}$ values.

\section{RESULTS}

A total of 217 patients who had AIS undergoing MT met the inclusion criteria, 164 (76.6\%, 164 of 217) of whom suffered from LVO in the anterior cerebral circulation and 
Table 1 Baseline characteristics of the study cohort

\begin{tabular}{|c|c|c|c|}
\hline & Total, $\mathrm{N}=164$ & $\mathrm{ICH}, \mathrm{n}=60$ & No ICH, $n=104$ \\
\hline Age (years) & $65(56-75)$ & $67(56-75)$ & $64(55-75)$ \\
\hline Female sex & $52(31.7)$ & $22(36.7)$ & $30(28.8)$ \\
\hline \multicolumn{4}{|l|}{ Comorbidities } \\
\hline Hypertension & $100(61.0)$ & $34(56.7)$ & $66(63.5)$ \\
\hline Diabetes mellitus & $69(42.1)$ & $30(50)$ & $39(37.5)$ \\
\hline Atrial fibrillation & $62(37.8)$ & $32(53.3)$ & $30(28.8)$ \\
\hline Current smoking & $71(43.3)$ & $24(40)$ & $47(45.2)$ \\
\hline Antiplatelet drug use & $20(12.2)$ & $8(13.3)$ & $12(11.5)$ \\
\hline Anticoagulant use & $12(7.3)$ & $5(8.3)$ & $7(6.7)$ \\
\hline \multicolumn{4}{|l|}{ Baseline laboratory values } \\
\hline Glucose, mg/dL & $6.9(5.5-9.8)$ & $7.8(6.1-10.2)$ & $6.6(5.1-8.6)$ \\
\hline Platelet count, $\times 109 / \mathrm{L}$ & $191(158-232)$ & $186(151-218)$ & $192(164-234)$ \\
\hline INR & $1.1(0.9-1.3)$ & $1.1(1.0-1.4)$ & $1.1(0.9-1.3)$ \\
\hline \multicolumn{4}{|l|}{ Stroke characteristics } \\
\hline Baseline NIHSS score & $13(10-17)$ & $14(12-18)$ & $12(9-16)$ \\
\hline Baseline GCS score & $13(9-15)$ & $12(9-14)$ & $13(10-15)$ \\
\hline ICA occlusion & $62(37.8)$ & $20(33.3)$ & $42(40.4)$ \\
\hline M1 occlusion & $96(58.5)$ & $59(98.3)$ & $37(35.6)$ \\
\hline M2 occlusion & $10(6.1)$ & $4(6.7)$ & $6(5.8)$ \\
\hline Left circulation & $84(51.2)$ & $30(50)$ & $54(51.9)$ \\
\hline \multicolumn{4}{|l|}{ Stroke treatment } \\
\hline Intravenous thrombolysis & $40(24.4)$ & $15(25)$ & $25(24)$ \\
\hline Onset-to-groin (min) & 327.5 (229.3-437.8) & $312.5(237.5-421.5)$ & $332.5(214.3-475.0)$ \\
\hline Procedure time (min) & $119.5(84-150)$ & $124(92.3-174.5)$ & $116(81.5-141.5)$ \\
\hline General anesthesia & $18(11.0)$ & $7(11.7)$ & $11(10.6)$ \\
\hline Conscious sedation & $146(89.0)$ & $53(88.3)$ & $93(89.4)$ \\
\hline $\mathrm{mTICl} 0$ & $15(9.1)$ & $8(13.3)$ & $7(6.7)$ \\
\hline $\mathrm{mTICl} 1$ & $4(2.4)$ & $2(3.3)$ & $2(1.9)$ \\
\hline $\mathrm{mTICl} 2 \mathrm{a}$ & $26(15.9)$ & $12(20)$ & $14(13.5)$ \\
\hline $\mathrm{mTICl} 2 \mathrm{~b}$ & $25(15.2)$ & $7(11.7)$ & $18(17.3)$ \\
\hline $\mathrm{mTICl} 3$ & $94(57.3)$ & $31(51.7)$ & $63(60.6)$ \\
\hline \multicolumn{4}{|c|}{ Blood pressure variables, $\mathrm{mm} \mathrm{Hg}$} \\
\hline Admission SBP & $146 \pm 24.3$ & $145 \pm 3.3$ & $147 \pm 2.5$ \\
\hline Admission DBP & $80 \pm 13.7$ & $81 \pm 1.8$ & $79 \pm 1.4$ \\
\hline Admission MAP & $102.0(92.7-109.6)$ & $102.3 \pm 2.0$ & $101.4 \pm 1.5$ \\
\hline \multicolumn{4}{|l|}{ Outcomes } \\
\hline Any ICH & $60(36.6)$ & & \\
\hline $\mathrm{HI}-1$ & $14(8.5)$ & & \\
\hline HI-2 & $17(10.4)$ & & \\
\hline $\mathrm{PH}-1$ & $14(8.5)$ & & \\
\hline $\mathrm{PH}-2$ & $15(9.1)$ & & \\
\hline
\end{tabular}

Variables are shown as mean $\pm \mathrm{SD}, \mathrm{n}(\%)$, or median and IQR.

DBP, diastolic blood pressure; GCS, Glasgow Coma Scale; HI, hemorrhagic infarction; ICA, internal carotid artery; ICH, intracerebral hemorrhage; INR, international normalized ratio; MAP, mean arterial pressure; mTICI, modified Thrombolysis in Cerebral Ischemia; NIHSS, National Institutes of Health Stroke Scale; PH, parenchymal hematoma; SBP, systolic blood pressure.

were included in this study. Among the enrolled subjects, 60 (36.6\%, 60 of 164) patients suffered from ICH during the first 24 hours after MT; HI-2 was the most common (10.4\%, 17 of 164), followed by PH-2 (9.1\%, 15 of 164), HI-1 (8.5\%, 14 of 164), and PH-1 (8.5\%, 14 of 164). Successful recanalization was achieved in 119 patients $(72.6 \%, 119$ of 164$)$ (mTICI $2 \mathrm{~b}-3)$, with a complete (mTICI 3) recanalization rate of $57.3 \%$ (94 of 164). The median age was $65(56-75)$ years and $31.7 \%$ (52 of 164) were female. The mean admission BP was $146 \pm 24 / 80 \pm 14 \mathrm{~mm} \mathrm{Hg}$. The baseline characteristics of the study cohort are detailed in table 1.

As shown in table 2, BP parameters were compared between patients with and without ICH. The maximum $(89.5 \mathrm{~mm} \mathrm{Hg}$ vs $98.5 \mathrm{~mm} \mathrm{Hg}, \mathrm{p}=0.001)$ and SD $(9.8 \mathrm{~mm}$ $\mathrm{Hg}$ vs $10.9 \mathrm{~mm} \mathrm{Hg}, \mathrm{p}=0.038$ ) of DBP during the first 24 hours of monitoring were higher in the group with ICH. Admission, mean, and range of BP did not significantly differ between groups with and without ICH. Multivariable logistic regression revealed that higher maximum DBP 
Table 2 Blood pressure variables during 24 hours following mechanical thrombectomy between patients with and without ICH

\begin{tabular}{|c|c|c|c|c|c|}
\hline \multirow[b]{2}{*}{ Variable } & \multirow[b]{2}{*}{ No ICH, $n=104$} & \multicolumn{3}{|l|}{$\mathrm{ICH}, \mathrm{n}=60$} & \multirow[b]{2}{*}{ Adjusted $p$ value } \\
\hline & & & $P$ value & Adjusted OR* $(95 \% \mathrm{Cl})$ & \\
\hline Admission SBP & $147(24)$ & $145(24)$ & 0.554 & - & - \\
\hline Admission DBP & $79(14)$ & $81(13)$ & 0.457 & - & - \\
\hline 24-hour SBP mean & $129(17)$ & $131(14)$ & 0.412 & - & - \\
\hline 24-hour DBP mean & $70(10)$ & $73(10)$ & 0.166 & - & - \\
\hline 24-hour MAP mean & $90(12)$ & $92(12)$ & 0.485 & - & - \\
\hline 24-hour SBP SD & $11.9(10-15)$ & $12.5(10-17)$ & 0.144 & - & - \\
\hline 24-hour DBP SD & $9.8(3.1)$ & $10.9(3.4)$ & 0.038 & $1.06(0.95$ to 1.18$)$ & 0.34 \\
\hline 24-hour SBP CV & $9.8(3.0)$ & $10.6(3.9)$ & 0.223 & - & - \\
\hline 24-hour DBP CV & $13.9(4.3)$ & $15.1(5.3)$ & 0.122 & - & - \\
\hline 24-hour SBP max & $154(22)$ & $160(19)$ & 0.071 & 1.01 (0.99 to 1.03$)$ & 0.19 \\
\hline 24-hour SBP min & $104(14)$ & $107(13)$ & 0.153 & - & - \\
\hline 24-hour DBP max & $89.5(83-100)$ & $98.5(93-107)$ & 0.001 & 1.03 (1.01 to 1.05$)$ & 0.028 \\
\hline 24-hour DBP min & $53(10)$ & $55(11)$ & 0.257 & - & - \\
\hline 24-hour SBP range & 47 (39-59.5) & $48(40-63)$ & 0.330 & - & - \\
\hline 24-hour DBP range & $39(14)$ & $43(15)$ & 0.055 & $1.02(0.99$ to 1.04$)$ & 0.14 \\
\hline
\end{tabular}

Variables are mean + SD or median and IQR.

${ }^{*}$ Adjusted for age, baseline NIHSS score, baseline GCS score, recanalization status (successful defined as mTICI $2 \mathrm{~b}-3$ vs unsuccessful defined as mTICI 0-2a) and procedure time. CV, coefficient of variance; DBP, diastolic blood pressure; GCS, Glasgow Coma Scale; ICH, intracerebral hemorrhage; MAP, mean arterial pressure; max, maximum; min, minimum; mTICl, modified Thrombolysis in Cerebral Ischemia; NIHSS, National Institutes of Health Stroke Scale; SBP, systolic blood pressure.

within 24 hours after MT was associated with higher odds of ICH (OR 1.03, 95\% CI 1.01 to $1.05, \mathrm{p}=0.028$ ) when adjusted for age, baseline NIHSS score, baseline GCS score, recanalization status (successful defined as mTICI $2 b-3$ vs unsuccessful defined as mTICI $0-2 a$ ) and procedure time.

Table 3 shows the optimal cut-off BP values to differentiate patients with versus without ICH. The optimal cut-off for maximum SBP was $155 \mathrm{~mm} \mathrm{Hg}$ (65\% sensitivity, 58\% specificity, area under the curve 0.59 , 95\% CI 0.50 to $0.68, \mathrm{p}=0.048$; figure 1$)$. A maximum SBP of $>155 \mathrm{~mm} \mathrm{Hg}$ yielded an unadjusted OR of 2.53 (1.31$4.89)(p=0.006)$ and an adjusted OR of $2.42(1.20-4.87)$ $(\mathrm{p}=0.013)$ for suffering ICH. For maximum DBP, an ideal cut-off value of $92.5 \mathrm{~mm} \mathrm{Hg}$ was identified $(80 \%$ sensitivity, $57 \%$ specificity, area under the curve 0.65 , 95\% CI 0.57 to $0.74, p=0.001$; figure 1$)$. A total of 93 patients had a maximum DBP $>92.5 \mathrm{~mm} \mathrm{Hg}$, of whom $48(80 \%)$ had ICH as compared with $12(17 \%)$ of 71 patients with maximum DBP $\leq 92.5 \mathrm{~mm} \mathrm{Hg}$. Patients with a maximum DBP $>92.5 \mathrm{~mm} \mathrm{Hg}$ had 4.56 times higher odds of ICH.

To explore the potential of recanalization status on the relationship between BP and ICH after MT, patients were divided into two subgroups of successful reperfusion (defined as mTICI 2b-3) and incomplete reperfusion (defined as mTICI 0-2a). Compared with the successful reperfusion group, the proportion of patients without $\mathrm{ICH}$ in the incomplete reperfusion group was lower $(51 \%$ vs
$68 \%$ ). In the subgroup of successful reperfusion, patients who had higher maximum SBP or maximum DBP within the first 24 hours after MT were more likely to experience ICH (mean for maximal SBP, 160 vs 152, $\mathrm{p}=0.037$; mean for maximal DBP, 98 vs 91, $\mathrm{p}=0.024$; table 4). However, there was no significant difference in maximal SBP and DBP in patients with incomplete reperfusion after MT. Of the 119 patients with successful reperfusion, a maximal SBP $>158.5 \mathrm{~mm} \mathrm{Hg}$ was associated with a higher rate of $\mathrm{ICH}$ in both unadjusted (OR $3.07(1.38-6.84) ; \mathrm{p}=0.006)$ and adjusted (OR $2.89(1.28-6.53) ; \mathrm{p}=0.011)$ analyses (online supplemental table 1 and figure 2). Similar outcomes were also found for maximal DBP $>91.5 \mathrm{~mm} \mathrm{Hg}$ (unadjusted OR 5.18 (2.12-12.7), p<0.001; adjusted OR 4.94 (1.9912.24), $\mathrm{p}=0.001$; online supplemental table 1 ).

\section{DISCUSSION}

In this study, we showed that higher maximal systemic arterial BP within the first 24 hours after MT was independently associated with a higher risk of ICH. In addition, patients with a maximal post-MT SBP $>155 \mathrm{~mm} \mathrm{Hg}$ or post-MT DBP $>92.5 \mathrm{~mm} \mathrm{Hg}$ have a higher likelihood of suffering ICH within 24 hours after MT. Subgroup analysis of patients with successful reperfusion (defined as mTICI 2b-3) also showed similar effects of higher BP on ICH. Therefore, the optimal management of both SBP and DBP

Table 3 Association of blood pressure with ICH

\begin{tabular}{|c|c|c|c|c|}
\hline Outcome & $\mathrm{OR}$ & $P$ value & Adjusted OR* $(95 \% \mathrm{Cl})$ & $\begin{array}{l}\text { Adjusted } \mathrm{p} \\
\text { value }\end{array}$ \\
\hline Any ICH (maximal SBP dichotomized >155 mm Hg) & $2.53(1.31-4.89)$ & 0.006 & 2.42 (1.20 to 4.87$)$ & 0.013 \\
\hline Any ICH (maximal SBP as continuous variable per $1 \mathrm{~mm} \mathrm{Hg}$ increase) & $1.01(1.00-1.03)$ & 0.073 & $1.01(1.00$ to 1.03$)$ & 0.19 \\
\hline Any ICH (maximal DBP dichotomized $>92.5 \mathrm{~mm} \mathrm{Hg}$ ) & $5.24(2.50-11.0)$ & $<0.001$ & $4.56(2.13$ to 9.76$)$ & $<0.001$ \\
\hline Any ICH (maximal DBP as continuous variable per $1 \mathrm{~mm} \mathrm{Hg}$ increase) & $1.03(1.01-1.06)$ & 0.009 & $1.03(1.01$ to 1.05$)$ & 0.028 \\
\hline
\end{tabular}

*Adjusted for age, baseline NIHSS score, baseline GCS score, recanalization status (successful defined as mTICI 2b-3 vs unsuccessful defined as mTICI 0-2a) and procedure time.

DBP, diastolic blood pressure; GCS, Glasgow Coma Scale; ICH, intracerebral hemorrhage; mTICI, modified Thrombolysis in Cerebral Ischemia; NIHSS, National Institutes of Health Stroke Scale; SBP, systolic blood pressure. 
A

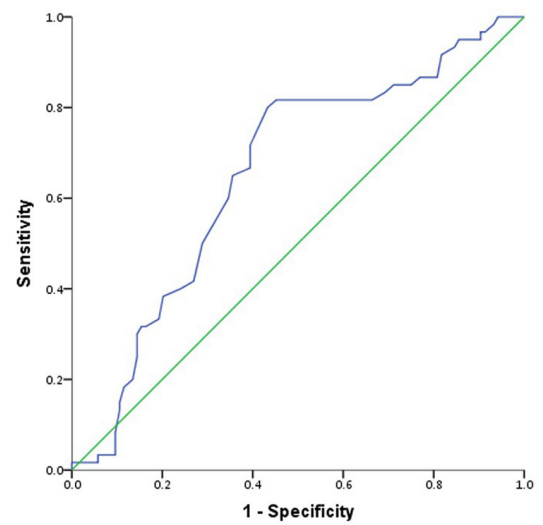

B

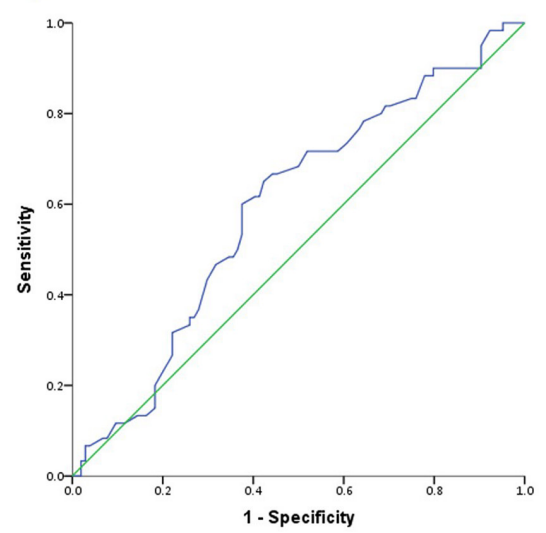

Figure 1 Receiver operating characteristic curves with corresponding AUC for maximum DBP (A) and SBP (B). The AUC of maximum DBP is $0.654(95 \% \mathrm{Cl} 0.567$ to $0.740, \mathrm{p}=0.001)$ and of maximum SBP $0.593(95 \% \mathrm{Cl} 0.504$ to $0.682, \mathrm{p}=0.048)$. AUC, area under the curve; DBP, diastolic blood pressure; SBP, systolic blood pressure.

should be taken seriously in patients who had AIS after MT to avoid ICH.

Previous studies found higher maximum SBP and SBP fluctuation after EVT to be associated with worse outcomes and higher rates of ICH in patients who had AIS. ${ }^{10} 13$ 17-19 The results of our study are in accordance with these prior studies. Moreover, maximal DBP within 24 hours after MT expressed a more significant effect on $\mathrm{ICH}$, but only few studies reported the association between DBP and outcomes in patients who had AIS after EVT. The resistance generated by peripheral arteries and arterioles forms the main part of DBP, and long-term hypertension can lead to progressive endothelial dysfunction, arterial stiffness and vasoconstriction of the arterioles until BP exceeds the upper limit of autoregulation, ${ }^{20} 21$ and which in turn causes vasodilation breakthrough, an increase in cerebral blood flow and blood-brain barrier dysfunction. ${ }^{22}$ Higher DBP indicates a decrease in large artery compliance. ${ }^{20} 23$ All the factors mentioned above can induce oxidative stress, inflammatory responses and other pathophysiological processes, causing ischemia-reperfusion injury in cerebral vessels. In this study, a peak DBP of $92.5 \mathrm{~mm} \mathrm{Hg}$ within 24 hours after MT best dichotomized ICH and no ICH. Patients with a peak DBP $>92.5 \mathrm{~mm} \mathrm{Hg}$ had 4.56 times higher odds of ICH. In our study, the stronger relationship between higher DBP and
ICH is an important finding, since many stroke physicians pay more attention to SBP to maintain it within safe ranges. The mean SBP was $131 \mathrm{~mm} \mathrm{Hg}$ in patients with $\mathrm{ICH}$ and $129 \mathrm{~mm} \mathrm{Hg}$ in patients without ICH in our study, whereas other studies reported a mean SBP of $159 \mathrm{~mm} \mathrm{Hg}$ in patients without ICH. ${ }^{13}$

Unlike DBP variables, there are vast data on the association between SBP in the first 24 hours post EVT and outcome, but there are less consistent results among these studies. A study of 228 patients with LVO in the anterior circulation demonstrated that both mean SBP and maximal SBP correlated with $\mathrm{ICH}$, including asymptomatic and symptomatic $\mathrm{ICH},{ }^{13}$ while other studies found no association between BP parameters and hemorrhagic complications. ${ }^{1024}$ The differences may be attributed to heterogeneity in inclusion criteria and the definition of hemorrhagic complications. Our study upheld that a maximal SBP of $155 \mathrm{~mm} \mathrm{Hg}$ and a maximal DBP of $92.5 \mathrm{~mm} \mathrm{Hg}$ in the 24 hours after MT best dichotomize ICH and no ICH. Patients in the maximal SBP $>155 \mathrm{~mm} \mathrm{Hg}$ group had 2.5 times higher odds of ICH as compared with patients whose maximal SBP was lower than $155 \mathrm{~mm} \mathrm{Hg}$.

A recent study in patients with successful revascularization after MT demonstrated that the course of elevated SBP and DBP was positively correlated with the rate of $\mathrm{ICH} .{ }^{12}$

Table 4 Association of maximal blood pressure as a continuous variable with $\mathrm{ICH}$ in the two subgroups of successful recanalization and incomplete recanalization

\begin{tabular}{|c|c|c|c|c|c|}
\hline \multicolumn{6}{|c|}{ Outcome with incomplete recanalization (mTICl 0-2a) } \\
\hline Variables, mean (SD) & $\begin{array}{l}\text { Any ICH } \\
n=22(49 \%)\end{array}$ & $\begin{array}{l}\text { No ICH } \\
n=23(51 \%)\end{array}$ & $P$ value & Adjusted $\mathrm{OR}^{*}(95 \% \mathrm{Cl})$ & Adjusted $\mathrm{p}$ value \\
\hline Maximal SBP as continuous & $160(17)$ & $162(26)$ & 0.788 & 1.00 (0.97 to 1.03$)$ & 0.730 \\
\hline Maximal DBP as continuous & $100(11)$ & $96(16)$ & 0.326 & 1.03 (0.98 to 1.08$)$ & 0.303 \\
\hline \multicolumn{6}{|c|}{ Outcome with successful recanalization ( $\mathrm{mTICl} 2 \mathrm{~b}-3)$} \\
\hline Variables, mean (SD) & $\begin{array}{l}\text { Any ICH } \\
n=38(32 \%)\end{array}$ & $\begin{array}{l}\text { No ICH } \\
n=81(68 \%)\end{array}$ & $P$ value & Adjusted OR* $(95 \% \mathrm{Cl})$ & Adjusted $\mathrm{p}$ value \\
\hline Maximal SBP as continuous & $160(20)$ & $152(21)$ & 0.037 & $1.02(1.00$ to 1.04$)$ & 0.054 \\
\hline Maximal DBP as continuous & $98(14)$ & $91(14)$ & 0.024 & 1.03 (1.01 to 1.06$)$ & 0.045 \\
\hline
\end{tabular}

Variables are mean \pm SD or $\mathrm{n}(\%)$

*Adjusted for age, baseline NIHSS score, baseline GCS score and procedure time

DBP, diastolic blood pressure; GCS, Glasgow Coma Scale; ICH, intracerebral hemorrhage; mTICI, modified Thrombolysis in Cerebral Ischemia; NIHSS, National Institutes of Health Stroke Scale; SBP, systolic blood pressure. 
A

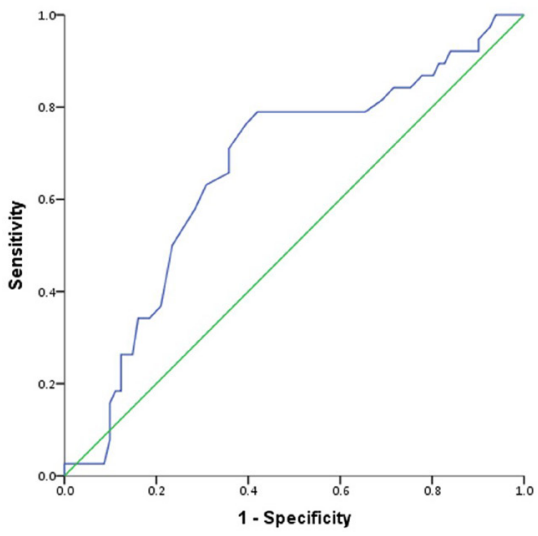

B

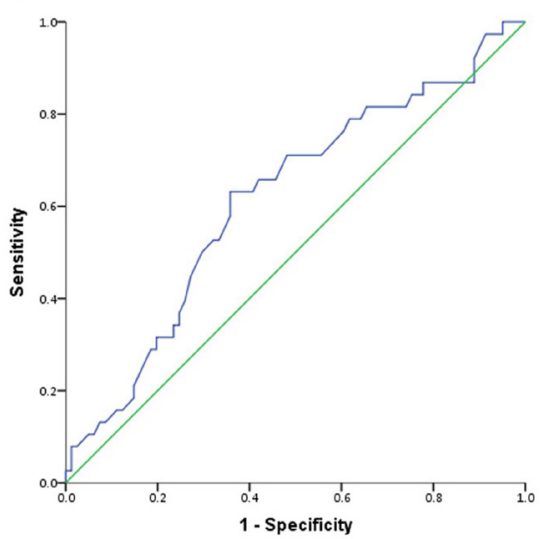

Another study in patients with non-recanalized LVO showed no relationship between the level of BP and symptomatic ICH. ${ }^{17}$ Given the different results by recanalization status, patients in our study were divided into two subgroups of successful reperfusion (mTICI 2b-3) and incomplete reperfusion (mTICI 0-2a). Interestingly, a similar result was also found in our study. Increased maximal SBP and maximal DBP yielded a significantly higher odds of $\mathrm{ICH}$ in the subgroup of successful reperfusion, but there was no such association in the subgroup of incomplete reperfusion. This may indicate that maximal BP within 24 hours after MT has a larger effect on patients with successful reperfusion.

\section{Limitations}

The presented study has some limitations. First, this was a retrospective study. Therefore, this study is associated with adherent bias and did not include long-term follow-up information. Second, due to the fact that the Heidelberg Bleeding Classification ${ }^{25}$ was not widely used in 2015, the classification of ICH was based on ECASS-II. Finally, the relatively small sample size limits the power for multivariable analysis.

\section{CONCLUSIONS}

Higher systemic arterial BP, especially maximal DBP, within 24 hours after MT in acute occlusions of the anterior cerebral circulation is associated with a greater risk of $\mathrm{ICH}$, regardless of age, admission NIHSS score, recanalization time and recanalization status.

\section{Author affiliations}

'Department of Neurology, The First Affiliated Hospital of Xi'an Jiaotong University, Xi'an, China

${ }^{2}$ Department of Cardiovascular Medicine, The Second Affiliated Hospital of Xi'an Jiaotong University, Xi'an, China

${ }^{3}$ Department of Cardiovascular Surgery, The First Affiliated Hospital of Xi'an Jiaotong University, Xi'an, China

${ }^{4}$ Department of Cardiology, University Heart Center Zürich, Zürich, Switzerland ${ }^{5}$ Department of Cardiovascular Medicine, The First Affiliated Hospital of Xi'an Jiaotong University, Xi'an, China

Acknowledgements We would like to thank all participating physicians and nurses.
Contributors JA contributed to conception and design of the work, drafting of the paper and interpretation of data. YT designed and collected the data. XC, HY, MW and XY contributed to acquisition of data. AZ, YL and AS revised the paper. GLi and GLo contributed to conception and design of the work and revised the paper. All authors read and approved the final manuscript.

Funding The authors have not declared a specific grant for this research from any funding agency in the public, commercial or not-for-profit sectors.

Competing interests None declared.

Patient consent for publication Not required.

Ethics approval This study was approved by the local institutional research and ethics committee (approval number XJTU1AF2018LSK-029).

Provenance and peer review Not commissioned; externally peer reviewed.

Data availability statement The data that support the findings of this study are available on request from the corresponding author (GLi).

Supplemental material This content has been supplied by the author(s). It has not been vetted by BMJ Publishing Group Limited (BMJ) and may not have been peer-reviewed. Any opinions or recommendations discussed are solely those of the author(s) and are not endorsed by BMJ. BMJ disclaims all liability and responsibility arising from any reliance placed on the content. Where the content includes any translated material, BMJ does not warrant the accuracy and reliability of the translations (including but not limited to local regulations, clinical guidelines, terminology, drug names and drug dosages), and is not responsible for any error and/or omissions arising from translation and adaptation or otherwise.

Open access This is an open access article distributed in accordance with the Creative Commons Attribution Non Commercial (CC BY-NC 4.0) license, which permits others to distribute, remix, adapt, build upon this work noncommercially, and license their derivative works on different terms, provided the original work is properly cited, an indication of whether changes were made, and the use is non-commercial. See: http://creativecommons.org/ licenses/by-nc/4.0/.

\section{ORCID iD}

Guoliang Li http://orcid.org/0000-0002-2352-9404

\section{REFERENCES}

1 Campbell BCV, Mitchell PJ, Kleinig TJ, et al. Endovascular therapy for ischemic stroke with perfusion-imaging selection. N Engl J Med 2015;372:1009-18.

2 Goyal M, Demchuk AM, Menon BK, et al. Randomized assessment of rapid endovascular treatment of ischemic stroke. N Engl J Med 2015;372:1019-30.

3 Jovin TG, Chamorro A, Cobo E, et al. Thrombectomy within 8 hours after symptom onset in ischemic stroke. N Engl J Med 2015;372:2296-306.

4 Saver JL, Goyal M, Bonafe A, et al. Stent-retriever thrombectomy after intravenous t-PA vs. t-PA alone in stroke. N Engl J Med 2015;372:2285-95. 
5 Bracard S, Ducrocq X, Mas JL, et al. Mechanical thrombectomy after intravenous alteplase versus alteplase alone after stroke (THRACE): a randomised controlled trial. Lancet Neurol 2016;15:1138-47.

6 Ragoschke-Schumm A, Walter S. Dawn and DEFUSE-3 trials: is time still important? Radiologe 2018;58:20-3.

7 Kaesmacher J, Kaesmacher M, Maegerlein C, et al. Hemorrhagic transformations after thrombectomy: risk factors and clinical relevance. Cerebrovasc Dis 2017;43:294-304.

8 Falcone GJ, Biffi A, Devan WJ, et al. Burden of blood pressure-related alleles is associated with larger hematoma volume and worse outcome in intracerebral hemorrhage. Stroke 2013;44:321-6.

9 Qureshi Al, Palesch YY, Barsan WG, et al. Intensive blood-pressure lowering in patients with acute cerebral hemorrhage. N Engl J Med 2016;375:1033-43.

10 Goyal N, Tsivgoulis G, Pandhi A, et al. Blood pressure levels post mechanical thrombectomy and outcomes in large vessel occlusion strokes. Neurology 2017:89:540-7.

11 Powers WJ, Rabinstein AA, Ackerson T, et al. 2018 guidelines for the early management of patients with acute ischemic stroke: a guideline for healthcare professionals from the American heart Association/American stroke association. Stroke 2018:49:e46-110.

12 Anadani M, Orabi MY, Alawieh A, et al. Blood pressure and outcome after mechanical thrombectomy with successful revascularization. Stroke 2019;50:2448-54.

13 Mistry EA, Mistry AM, Nakawah MO, et al. Systolic Blood Pressure Within 24 Hours After Thrombectomy for Acute Ischemic Stroke Correlates With Outcome. J Am Heart Assoc 2017;6. doi:10.1161/JAHA.117.006167. [Epub ahead of print: 18 May 2017].

14 Anadani M, Arthur AS, Alawieh A, et al. Blood pressure reduction and outcome after endovascular therapy with successful reperfusion: a multicenter study. J Neurointerv Surg 2020;12:932-6.
15 Delgado-Mederos R, Ribo M, Rovira A, et al. Prognostic significance of blood pressure variability after thrombolysis in acute stroke. Neurology 2008;71:552-8.

16 Larrue $V$, von Kummer R R, Müller A, et al. Risk factors for severe hemorrhagic transformation in ischemic stroke patients treated with recombinant tissue plasminogen activator: a secondary analysis of the European-Australasian acute stroke study (ECASS II). Stroke 2001;32:438-41.

17 Goyal N, Tsivgoulis G, Pandhi A, et al. Blood pressure levels post mechanical thrombectomy and outcomes in non-recanalized large vessel occlusion patients. J Neurointerv Surg 2018;10:925-31.

18 Kim TJ, Park H-K, Kim J-M, et al. Blood pressure variability and hemorrhagic transformation in patients with successful recanalization after endovascular recanalization therapy: a retrospective observational study. Ann Neurol 2019;85:574-81.

19 Mistry EA, Sucharew H, Mistry AM, et al. Blood pressure after endovascular therapy for ischemic stroke (best): a multicenter prospective cohort study. Stroke 2019;50:3449-55.

20 Rizzoni D, Rizzoni M, Nardin M, et al. Vascular aging and disease of the small vessels. High Blood Press Cardiovasc Prev 2019;26:183-9.

21 Qureshi Al. Acute hypertensive response in patients with stroke: pathophysiology and management. Circulation 2008;118:176-87.

22 Olsen TS, Larsen B, Herning M, et al. Blood flow and vascular reactivity in collaterally perfused brain tissue. Evidence of an ischemic penumbra in patients with acute stroke. Stroke 1983;14:332-41.

23 Nilsson PM, Boutouyrie P, Laurent S. Vascular aging: a tale of EVA and ADAM in cardiovascular risk assessment and prevention. Hypertension 2009;54:3-10.

24 Anadani M, Orabi Y, Alawieh A, et al. Blood pressure and outcome post mechanical thrombectomy. J Clin Neurosci 2019;62:94-9.

25 von Kummer R, Broderick JP, Campbell BCV, et al. The Heidelberg bleeding classification: classification of bleeding events after ischemic stroke and reperfusion therapy. Stroke 2015;46:2981-6. 